\title{
Resident physician and hospital pharmacist familiarity with patient discharge medication costs
}

\author{
Kerry Wilbur
}

Received: 21 August 2008/Accepted: 28 December 2008/Published online: 29 January 2009

(C) Springer Science+Business Media B.V. 2009

\begin{abstract}
Objective Cost-related medication non-adherence is associated with increased health-care resource utilization and poor patient outcomes. Physicians-in-training generally receive little education regarding costs of prescribed therapy and may rely on hospital pharmacists for this information. However, little is documented regarding either of these health care providers' familiarity with out-of pocket medication expenses borne by patients in the community. The purpose of this study was to evaluate and compare resident physician and hospital pharmacist familiarity with what patients pay for medications prescribed once discharged. Setting A major tertiary patient care and medical teaching centre in Canada. Method Internal medicine residents and hospital pharmacists within a specific health care organization were invited to participate in an online survey. Eight patient case scenarios and associated discharge therapeutic regimens were outlined and respondents asked to identify the costs patients would incur when having the prescription filled once discharged. Main Outcome Measure Total number and proportion of estimates above and below actual cost were calculated and compared between the groups using $\chi^{2}$ tests. Responses $\pm 10 \%$ of the true cost were considered correct. Mean absolute values and standard deviation estimated costs, as well as cost increments above and below $10 \%$, were calculated to assess the magnitude of the discrepancy between the respondent estimates and the actual total cost. Results Forty-four percent of physician residents and $26 \%$ of hospital pharmacists accessed the survey. Overall $39 \%$ and $47 \%$ of medication costs were
\end{abstract}

\footnotetext{
K. Wilbur ( $₫)$

College of Pharmacy, Qatar University,

PO Box 2713, Doha, Qatar

e-mail: kwilbur@qu.edu.qa
}

under-estimated, 32\% and 33\% were overestimated, and $29 \%$ and $21 \%$ were correctly estimated by residents and pharmacists, respectively $(P=\mathrm{NS})$. Incorrect estimates were evident across all therapeutic classes and medical indications presented in the survey. The greatest absolute cost discrepancy for both groups was under-estimation of linezolid (\$800 and \$400) and over-estimation of clopidogrel (\$80) and bisoprolol therapy (\$22) by residents and pharmacists, respectively. Conclusion Resident physicians and hospital pharmacists are unfamiliar with what patients must pay for drug therapy once discharged.

Keywords Canada - Costs - Discharge medication . Pharmacist · Resident physician

\section{Impact statement of research findings on practice:}

- Drug costs are a barrier to medication adherence for many patients and can be a source of failed therapeutic outcomes.

- Residents and physicians are often unfamiliar with the cost of their prescribed therapy.

- Hospital pharmacists and resident physicians similarly lack an appreciation of medication costs.

\section{Introduction}

Prescription drug spending continues to outpace every other segment in health care as medications become more expensive, prescribing preferences shift to novel and more costly therapies and medication use rises with the aging population [1]. 
A common measure to control drug expenditures is implementation of cost-sharing strategies such as user fees that require consumers to assume a portion of prescription cost. As health care costs escalate, employers are increasingly shifting the proportion of drug costs they bear to the individual. Despite private or public drug benefit coverage, patients may be subject to higher co-payments, deductibles, as well as reimbursement and formulary restrictions [2, 3].

Drug therapy poses a financial burden for many patients and those who face high out-of-pocket medication expenses are less likely to purchase prescribed therapy [4-6]. Similarly, these patients may resort to administration minimization strategies such as inappropriate alternate day dosing or as needed use to decrease medication expenditures $[7,8]$. Inability of individuals to pay for medication ultimately increases the cost to health care systems [9, 10]. Consequences of cost-related non-adherence include increased use of emergency department and health care services as well as reduction in overall health status and are virtually always attributed to patients foregoing essential drug therapy [2, 11-15].

Health professionals developing discharge medication regimens from in-patient care settings may not always be aware of patients' specific drug therapy coverage or appreciate prescription costs in the community. It has been previously found that physicians do not routinely ask if patients have possible medication payment difficulties and are often uninformed about the costs associated with their prescribed therapy [2, 16-20]. Physicians-in-training (students, interns, residents) generally receive limited formal education regarding costs of medical care, including prescriptions [21]. In a teaching hospital, resident physicians may rely on pharmacist members of the multi-disciplinary team for this information. However, there is surprisingly little information regarding hospital pharmacists' knowledge of patient medication costs in the community. Pharmacists unacquainted with specific discrepancies between their hospital formulary systems and outpatient medication reimbursement structures forfeit the opportunity to recommend more affordable therapeutic alternatives to resident physicians prior to patient discharge. Therapeutic regimens initiated in hospital may then be interrupted due to financial hardship and patient outcomes compromised [22].

\section{Aim of the study}

The purpose of this study was to evaluate and compare medical resident physician and hospital pharmacist familiarity with how much patients pay for medications prescribed once discharged.

\section{Method}

In May 2007, internal medicine residents training and hospital pharmacists employed within four hospitals within a principle health authority in British Columbia, Canada were contacted by email and invited to participate in an online survey.

Subsequent survey questions presented eight brief patient case scenarios describing six typical reasons for hospital admission to the internal medicine service at the major tertiary care centre within this health authority according to hospital census data. Specific discharge therapeutic regimens were outlined with each patient case scenario and the resident physicians and pharmacists asked to estimate the associated discharge cost. These regimens were selected in accordance with best medical practice according to established clinical guidelines or likely microbiological data (e.g. the infectious diseases-related cases). Respondents were instructed to consider both direct drug costs and pharmacist professional fee when approximating the total cost a patient would pay at a local community pharmacy. It was identified in each case that patients had no third party coverage and would therefore incur the total cost of therapy themselves and that generic medication would be dispensed when available for alternatives illustrated in the scenarios.

The survey took approximately $20 \mathrm{~min}$ to complete. Two subsequent reminder messages were sent at 3-week intervals to encourage resident physicians and pharmacists to complete the online survey.

Actual costs of the discharge therapeutic regimens described in the patient case scenarios were obtained from three Greater Vancouver community pharmacies representing three different drug store chains. The mean value of each reported medication cost was used to compare with those costs estimated by survey respondents. All prices are reported in Canadian dollars.

Each drug regimen cost estimate was compared to the true cost and the absolute error (true cost minus estimate) calculated. The total number and proportion of resident physician and pharmacist estimates that were either above or below the actual cost were calculated. Responses within $10 \%$ of the actual total cost were considered correct. Mean absolute values and standard deviation of estimated costs, as well as cost increments above and below $10 \%$, were calculated to permit assessment of the magnitude of the discrepancy between the respondent estimates and the actual total cost. Correct price interval estimates were coded with a value of zero and the range of incorrect estimates were coded as the absolute value of the number of $10 \%$ intervals above or below the correct interval. Mean scores closer to zero therefore indicate greater familiarity with discharge regimen price. Incomplete surveys were 
included in the analysis and proportional data were expressed in terms of the number of respondents who answered a particular question.

All data analysis was conducted using SPSS for Windows standard version release 16.0 Excel (SPSS Inc.,Chicago, Illinois). Categorical data are presented as percentages of frequency or occurrence. Continuous data are presented as means with standard deviations (SD). The two groups' responses were compared using Chi-square tests. All statistical tests were 2-tailed and based on a significance level of $\alpha \leq 0.05$.

\section{Results}

The email invitation was distributed to the 96 physicians in the internal medicine residency training program and 146 hospital pharmacists, of whom 25 (26\%) and 64 (43.8\%), respectively accessed the online survey to participate.
For the total 29 alternative discharge regimens described in the six patient case scenarios, $39 \%$ and $46 \%$ of medication costs were under-estimated, $32 \%$ and $33 \%$ were over-estimated, and $29 \%$ and $21 \%$ were correctly estimated by resident physicians and pharmacists, respectively (Fig. 1).

Nearly half of all alternative discharge antibiotic therapy costs were under-estimated by both groups, but more so by pharmacists $(36.0 \%$ and $44.4 \%, P=0.047)$. Resident physician and pharmacist overall estimates of discharge medication costs for treatment of community-acquired pneumonia or skin and soft tissue infections described in the patient case scenarios were similarly incorrect. Specifically, most resident physician respondents underestimated cefuroxime and clarithromycin combination therapy and over-estimated moxifloxacin discharge regimens prescribed for pneumonia. Conversely, pharmacists were more likely to under-estimate the price of cefuroxime and over-estimate the price of azithromycin. Linezolid

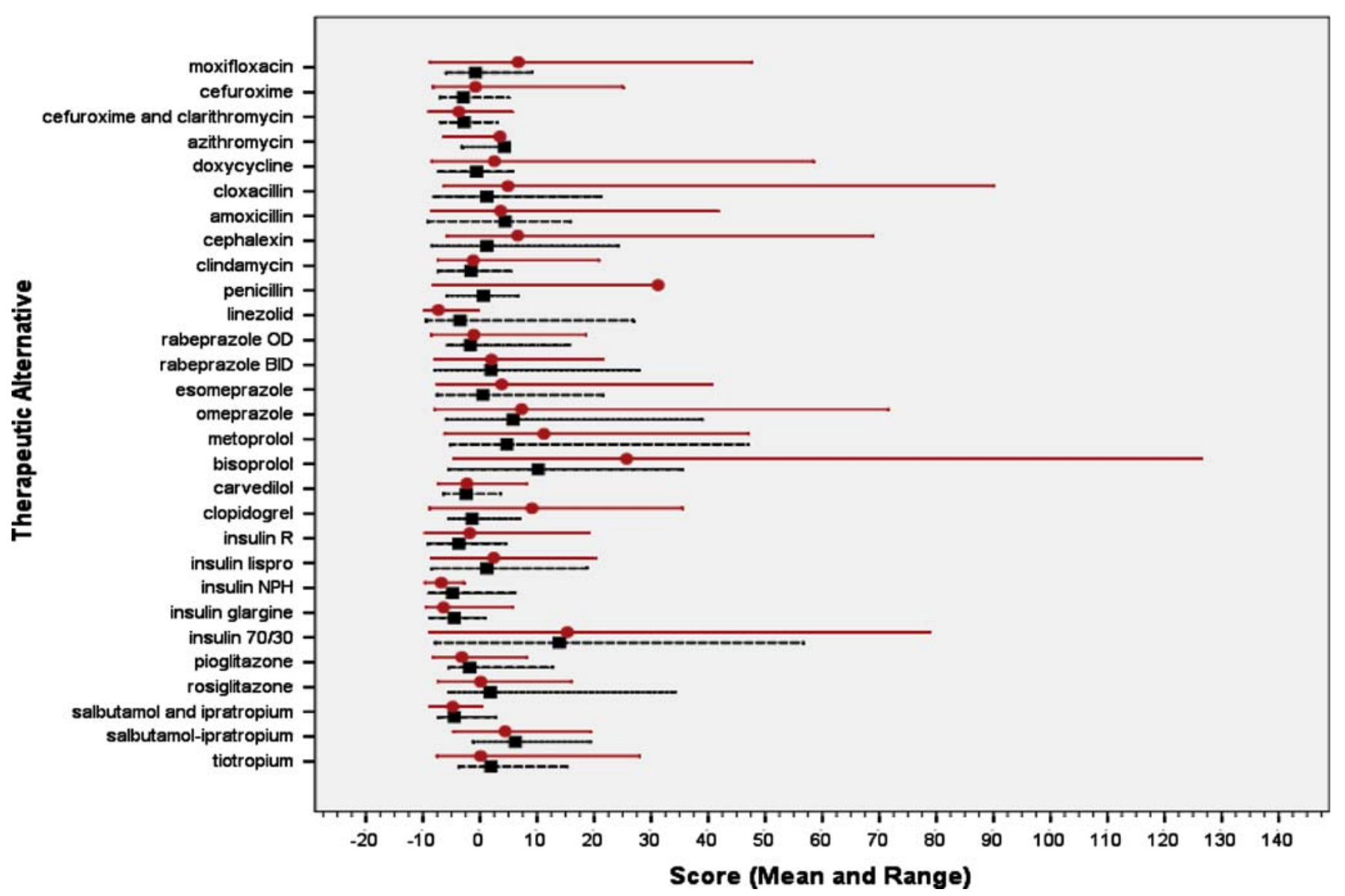

Fig. 1 Resident physician and hospital pharmacists estimates of discharge medication costs (Medication described on the $y$-axis represent therapeutic alternatives offered in the survey patient case scenarios. Pharmacists' cost estimates of medication regimens were considered correct if they fell within $\pm 10 \%$ of the actual cost. Actual medication regimen costs $( \pm 10 \%)$ were coded as zero. Therefore, mean scores closer to zero indicate greater familiarity with the cost of medication regimens. The range of cost estimates greater or less than $10 \%$ of the actual cost are also depicted. Each unit on the $x$-axis represents one incorrect cost interval $( \pm 10 \%$ of actual cost). Resident physician responses are illustrated in red.) 
therapy was most commonly under-estimated by both groups, but to a greater extent by resident physicians, while clindamycin and amoxicillin over-estimated by resident physician and pharmacists, respectively, when treatment of skin and soft tissue infection was considered.

Estimates of discharge proton pump inhibitor costs for peptic ulcer disease were similarly under- $(35.2 \%$ vs. $40.2 \%, \mathrm{NS})$ and over-estimated $(51.1 \%$ vs. $41.1 \%, \mathrm{NS})$ by resident physician and pharmacist respondents.

The discharge cost of carvedilol was most frequently under-estimated and that of bisoprolol over-estimated when beta blocker heart failure therapy scenarios were assessed. Likewise, all respondents were unable to accurately determine the cost of clopidogrel antiplatelet therapy, although it was were more likely to be over-estimated by resident physicians $(52.4 \%$ vs. $8.3 \%, P=0.001)$ and under-estimated by pharmacists $(10.1 \%$ vs. $69.4 \%$, $P=0.001$ ).

Respondents largely under-estimated community pharmacy expenses for diabetes treatment, including various insulin formulations $(57.1 \%$ vs. $54.4 \%, P=\mathrm{NS})$ and thiazolidinediones $(57.5 \%$ vs. $54.2 \%, P=\mathrm{NS})$.

Assessment of chronic obstructive pulmonary disease (COPD) therapy was also incorrect as many respondents under-estimated the price of salbutamol and ipratropium metered dose inhalers (MDI) $(95.4 \%$ vs. $91.3 \%, P=\mathrm{NS})$, but over-estimated the cost of the combination product (Combivent $\left.{ }^{\circledR}\right)(68.2 \%$ vs. $65.2 \%, P=\mathrm{NS})$.

When considering mean absolute costs, the greatest under-estimation among both groups was of linezolid therapy by an average of $\$ 800$ for resident physicians and nearly $\$ 400$ for pharmacists. Resident physicians overestimated clopidogrel by $\$ 80$ and pharmacist over-estimation of bisoprolol therapy was by approximately $\$ 20$ (Table 1). However, errors as a proportion of actual cost $( \pm 10 \%)$ were most often found among insulin and cardiovascular therapies: NPH ( -6.8 and -4.3$)$ and insulin 70/30 (13.9) and bisoprolol (25.7) by pharmacists and resident physicians, respectively (Fig. 1).

\section{Discussion}

Both internal medicine resident physicians and hospital pharmacists demonstrated poor knowledge of discharge medication costs. Incorrect estimates were evident across the spectrum of therapeutic classes and associated medical indications presented in the survey. The expense borne by patients at discharge was underestimated for half of the proposed therapeutic regimens ranging from a few to hundreds of dollars.

Patients are frequently discharged with new drug therapies and more complex and costly regimens following hospital stays [23, 24]. The economic factors of polypharmacy, coupled with competing demands for household resources may prevent patients from completing or sustaining intended therapy [25]. Cost-related decreases in prescription medication use invariably impacts those most vulnerable to poor outcome: the poor; the elderly; and the chronically ill [2].

Our results reflect physician shortcomings in anticipating patient medication costs that are well established, but less well-documented for physicians-in-training [16-21]. Existing data suggests they also have poor cost awareness, undoubtedly linked to inexperience, as well as insufficient information within medical education curricula [21]. Family practice residents previously surveyed in British Columbia paradoxically overestimated the costs of inexpensive drugs and underestimated the costs of expensive drugs [26]. In fact, some expressed frustration about their ignorance and refused to participate because of it. However, when critical care staff were surveyed in another study, professional experience (staff versus resident) had no impact on discouraging cost estimate performance [27]. Physicians-in-training invariably receive informal teaching from multidisciplinary team members during their practice rotations, so it is particularly unfortunate and surprising for hospital pharmacists to demonstrate inadequate knowledge of drug prices. In one physician survey, over $50 \%$ believed that pharmacists have a greater responsibility for managing patients' out-of-pocket costs than doctors [19]. Clearly, physicians rely on pharmacists to inform patients of drug therapy costs and intervene if possible when financial obstacles are identified. Hospital pharmacists are responsible for guiding all health staff involved in discharge planning to ensure optimal medication outcomes for patients, but this support is especially important in teaching hospitals where novice prescribers are faced with other patient care demands and stresses [28].

Hospital pharmacists should make an overt inquiry regarding prescription drug coverage a routine part of patient interactions. It has been previously demonstrated that individuals are not necessarily forthcoming with inabilities to support the costs of medication but expressed a preference to discuss strategies to manage these expenses with their health care provider [29, 30]. However, patients may not be able to anticipate difficulties obtaining discharge therapy if they are not advised of the potential cost to fill prescriptions in the community. For this reason, both resident physicians and hospital pharmacists need to familiarize themselves and keep abreast with the costs of the most common discharge therapies in their clinical practice and any restrictive or supportive governmental policies concerning specific patient or medication coverage.

It is not with the greatest ease that community physicians or pharmacists can coordinate medication coverage 
Table 1 Hospital pharmacists' estimates of discharge medication costs

\begin{tabular}{|c|c|c|c|}
\hline Discharge drug regimen ${ }^{\mathrm{a}}$ & Actual $\cos \mathrm{t}^{\mathrm{b}}$ & $\begin{array}{l}\text { Resident physician } \\
\text { mean discrepancy }(\mathrm{SD})^{\mathrm{c}}\end{array}$ & $\begin{array}{l}\text { Hospital pharmacist } \\
\text { mean discrepancy }(\mathrm{SD})^{\mathrm{c}}\end{array}$ \\
\hline \multicolumn{4}{|l|}{ Community-acquired pneumonia } \\
\hline Moxifloxacin $400 \mathrm{mg}$ po daily & $\$ 51.98$ & $\$ 32.73(\$ 68.89)$ & $\$-3.93(\$ 16.38)$ \\
\hline Cefuroxime $500 \mathrm{mg}$ po BID & $\$ 39.99$ & $\$-3.23(\$ 28.24)$ & $\$-11.79(\$ 11.69)$ \\
\hline Cefuroxime + clarithromycin $500 \mathrm{mg}$ po BID & $\$ 95.90$ & $\$-31.73(\$ 40.17)$ & $\$-25.55(\$ 27.58)$ \\
\hline Azithromycin $250 \mathrm{mg}$ po daily (5 days) & $\$ 27.94$ & $\$ 9.32(\$ 26.85)$ & $\$ 12.13(\$ 15.12)$ \\
\hline Doxycyline $100 \mathrm{mg}$ po daily & $\$ 19.10$ & $\$ 4.77(\$ 25.83)$ & $\$-1.17(\$ 6.90)$ \\
\hline \multicolumn{4}{|l|}{ Skin and soft tissue infection } \\
\hline Amoxicillin/clavulanate $875 \mathrm{mg}$ po BID & $\$ 29.28$ & $\$ 4.77(\$ 25.83)$ & $\$ 12.82(\$ 17.24)$ \\
\hline Cephalexin $500 \mathrm{mg}$ po QID & $\$ 19.21$ & $\$ 12.55(\$ 30.25)$ & $\$ 2.25(\$ 13.46)$ \\
\hline Clindamycin $450 \mathrm{mg}$ po QID & $\$ 39.39$ & $\$ 18.18(\$ 31.36)$ & $\$-5.89(\$ 14.09)$ \\
\hline Linezolid $600 \mathrm{mg}$ po BID & $\$ 1,088.08$ & $-\$ 782.50(\$ 326.98)$ & $\$-384.18(\$ 757.01)$ \\
\hline Penicillin VK 500 mg po QID & $\$ 11.99$ & $\$ 37.45(\$ 103.74)$ & $\$ 1.00(\$ 4.74)$ \\
\hline \multicolumn{4}{|l|}{ Peptic ulcer disease } \\
\hline Rapebrazole $20 \mathrm{mg}$ po daily $\times 30$ days & $\$ 70.31$ & $\$-7.68(\$ 45.67)$ & $-\$ 11.60(\$ 31.10)$ \\
\hline Rabeprazole $20 \mathrm{mg}$ po bid $\times 30$ days & $\$ 95.33$ & $\$ 20.05(\$ 75.58)$ & $\$ 18.60(\$ 64.19)$ \\
\hline Esomeprazole $20 \mathrm{mg}$ po daily $\times 30$ days & $\$ 78.61$ & $\$ 29.86(\$ 109.80)$ & $\$ 3.96(\$ 42.91)$ \\
\hline Omeprazole $20 \mathrm{mg}$ po daily $\times 30$ days & $\$ 49.30$ & $\$ 35.68(\$ 95.63)$ & $\$ 26.08(\$ 45.90)$ \\
\hline \multicolumn{4}{|l|}{ Heart failure—beta blocker } \\
\hline Metoprolol $50 \mathrm{mg}$ po BID & $\$ 21.05$ & $\$ 24.50(\$ 36.18)$ & \$9.96 (\$22.14) \\
\hline Bisoprolol $10 \mathrm{mg}$ po daily & $\$ 21.92$ & $\$ 52.09(\$ 54.59)$ & $\$ 22.42(\$ 20.48)$ \\
\hline Carvedilol $50 \mathrm{mg}$ po BID & $\$ 109.55$ & $-\$ 25.09(\$ 48.59)$ & $\$-26.88(\$ 35.64)$ \\
\hline \multicolumn{4}{|l|}{ Cardiovascular disease-Antiplatelet } \\
\hline Clopidogrel $75 \mathrm{mg}$ po daily & $\$ 88.15$ & $\$ 80.33(\$ 223.30)$ & $\$-12.00(\$ 23.00)$ \\
\hline \multicolumn{4}{|l|}{ Diabetes-Insulin } \\
\hline NPH (5 vials) & $\$ 104.71$ & $\$-71.90(\$ 22.81)$ & $\$-50.40(\$ 42.24)$ \\
\hline Glargline (5 vials) & $\$ 319.13$ & $\$-204.28(\$ 135.83)$ & $\$-143.80(\$ 109.14)$ \\
\hline REGULAR (1 vial) & $\$ 24.33$ & $\$-4.35(\$ 19.38)$ & $\$-8.84(\$ 8.15)$ \\
\hline Lispro (1 vial) & $\$ 32.91$ & $\$ 4.55(\$ 33.73)$ & $\$ 4.08(\$ 18.47)$ \\
\hline 70/30 pen ( 5 cartridges) & $\$ 45.26$ & $\$ 14.20(\$ 21.81)$ & $\$ 12.52(\$ 13.95)$ \\
\hline \multicolumn{4}{|l|}{ Diabetes-Thiazolidinedione } \\
\hline Pioglitazone $30 \mathrm{mg}$ po daily $\times 30$ days & $\$ 109.33$ & $\$-35.33(\$ 46.99)$ & $\$-20.00(\$ 47.41)$ \\
\hline Rosiglitazone $4 \mathrm{mg}$ po daily $\times 30$ days & $\$ 77.18$ & $\$ 0.86(\$ 51.65)$ & $\$ 14.46(\$ 60.48)$ \\
\hline \multicolumn{4}{|l|}{ Chronic obstructive pulmonary disease } \\
\hline Salbutamol and ipratropium (2 MDI each) & $\$ 77.81$ & $\$-37.64(\$ 19.01)$ & $\$-34.96(\$ 19.99)$ \\
\hline Salbutamol with ipratropium (1 MDI) & $\$ 33.98$ & $\$ 15.00(\$ 22.87)$ & $\$ 21.22(\$ 23.05)$ \\
\hline Tiotropium (30 capsules) & $\$ 79.26$ & $\$ 0.95(\$ 58.10)$ & $\$ 14.65(\$ 41.06)$ \\
\hline
\end{tabular}

${ }^{\text {a }}$ Respondents $(n=31)$ were asked to estimate the cost of one month's supply prescribed at hospital discharge

b Actual costs are in Canadian dollars and represent the mean price of the regimen dispensed from three different British Columbia community pharmacies

c The mean value of pharmacists' estimates of a medication regimen is compared to the actual cost and the discrepancy reported as an absolute dollar value $(\mathrm{SD}=$ standard deviation)

or therapeutic substitution. Hospital pharmacists are in a unique position to make timely interventions in the selection of the most appropriate and affordable therapeutic regimen prior to patient discharge. Gaps in seamless care is a disservice and inconvenience to both patients and community pharmacist colleagues whose ability to advocate for less expensive therapies is often restricted by resources and information. Indeed, while physicians feel pharmacists shoulder greater responsibility for mediating prescription costs for patients, over half responding to one survey felt that phone calls from pharmacists were "bothersome" [19]. 
Adequate evaluation of patients' ability to support medication costs may be limited by patient volume and increasingly rapid or abrupt hospital discharges. To overcome these barriers, certain organizations have implemented devoted pharmacist- or nurse-run discharge services that have improved the efficiency and quality of prescription decision-making in the discharge planning process [31-33]. Activities include accommodating more appropriate therapeutic selections according to patient drug benefit plans and other financial considerations. Swift and efficient access to community drug pricing through various technological mediums would further facilitate responsible discharge planning. A number of states in the US support websites with prescription drug cost search engines or "price finders" accessible to both patients and health-care providers [34, 35]. Hospital medication inventory software could also be programmed with community drug costs and linked to inpatient medication profiles. Physician and students alike express a desire for more cost information to consider in medical-decision making processes to reduce costs to patients and the health care system [20, 21]. Personalized physician feedback has been demonstrated to alter prescribing patterns and reduce costs in other studies [20].

While this appears to be the first survey of its kind, there are a number of limitations which warrant discussion. The low response rate restricts generalizability to the hospital pharmacist workforce and resident physicians training within this health authority and indeed beyond. The patient and therapeutic scenarios described in the survey were drawn from general internal medicine cases; respondents were not asked if they had been involved in the discharge planning of actual patients receiving these specific therapies and may already be familiar with their cost. Survey responses would not necessarily reflect accurate discharge drug cost knowledge of hospital pharmacists who provide clinical services in different patient care settings or specialties. The survey also did not identify whether hospital pharmacist respondents worked in community pharmacy practice and had dispensed these specific therapies or otherwise had first-hand knowledge of their price. The actual costs of therapeutic regimens outlined in the patient case scenarios were drawn from only three community pharmacies. Despite the $10 \%$ margin for correct estimation, it is conceivable that actual medication costs may have in fact varied more widely if data was reported from other community pharmacy practices and would therefore alter the proportion of over-and under-estimations. It is expected that junior resident respondents would be less aware of prescription costs than their senior resident peers. Finally, incorrect estimate of drug therapy costs when prompted by a questionnaire should not imply resident physicians or pharmacists do not appropriately investigate the discharge costs for patients at the point of hospital discharge.

\section{Conclusion}

Resident physicians and hospital pharmacists were unfamiliar with what internal medicine patients must pay for prescribed discharge therapy once they have returned to the community. More studies are warranted to further characterize this problem as simple strategies exist to avoid this ameliorable source for medication non-adherence.

Acknowledgements All pharmacists and resident physicians who participated in the survey.

Funding No special funding was obtained for this study.

Conflict of interest The author has no conflicts of interest to declare.

\section{References}

1. Canadian Institute for Health Information. Drug Expenditure in Canada 1985-2007. Ottawa: CIHI; 2008.

2. Lexchin J, Grootendorst P. Effects of prescription drug user fees on drug and health services use and on health status in vulnerable populations: a systematic review of the evidence. Int $\mathrm{J}$ Health Serv. 2004;34(1):101-22. doi:10.2190/4M3E-L0YF-W1TDEKG0.

3. Grootendorst P. Beneficiary cost sharing under Canadian provincial prescription drug benefit programs: history and assessment. Can J Clin Pharmacol. 2002;9(2):79-99.

4. Leibowitz A, Manning WG, Newhouse JP. The demand for prescription drugs as a function of cost-sharing. Soc Sci Med. 1985;21(10):1063-9. doi:10.1016/0277-9536(85)90161-3.

5. Soumerai SB, Pierre-Jacques M, Zhang F, Ross-Degnan D, Adams AS, Gurwitz J, et al. Cost-related medicaton non-adherence among elderly and disabled medicare beneficiaries. A national survey 1 year before the medicare drug benefit. Arch Intern Med. 2006;166(17):1829-35. doi:10.1001/archinte.166. 17.1829.

6. Lillard La, Rogowski J, Kingston R. Insurance coverage for prescription drugs: effects on use and expenditures in the medicare population. Med Care. 1999;37(9):926-36. doi:10.1097/00 005650-199909000-00008.

7. Steinman M, Sands LP, Covinsky KE. Self-restriction of medications due to cost in seniors without prescription coverage. A national survey. J Gen Intern Med. 2001;16(12):793-9. doi: 10.1046/j.1525-1497.2001.10412.x.

8. Piette JD, Heisler M, Wagner TH. Cost-related medication underuse. Do patients with chronic illness tell their doctors? Arch Intern Med. 2004;164(16):1749-55. doi:10.1001/archinte.164. 16.1749 .

9. Urquhart J. Pharmacoeconomic consequences of variable patient compliance with prescribed drug regimens. Pharmacoeconomics. 1999;15(3):317-28. doi:10.2165/00019053-199915030-00002.

10. Cleemput I, Kesteloot K. Economic implications of non-compliance in health care. Lancet. 2002;359(9324):2129-30. doi: 10.1016/S0140-6736(02)09114-6.

11. Tamblyn R, Laprise R, Hanley JA, Abrahamowicz M, Scott S, Mayo N, et al. Adverse events associated with prescription drug cost-sharing among poor and elderly persons. JAMA. 2001;285(4): 421-9. doi:10.1001/jama.285.4.421. 
12. Soumerai SB, Ross-Degnan D, Avorn J, McLaughlin TJ, Choodnovskkiy I. Effects of medicaid drug-payment limits on admission to hospitals and nursing homes. N Engl J Med. 1991; 325(15):1072-7.

13. Russell LB, Wolff N. The impact of drug pricing policies on the health of the elderly. Am J Prev Med. 2002;22(3):151-5. doi: 10.1016/S0749-3797(01)00432-9.

14. Anis AH, Guh DP, Lacaille D, Marra CA, Rashidi AA, Lix E, et al. When patients have to pay a share of drug costs. Effects on frequency of physician visits, hospital admissions and filling of prescriptions. CMAJ. 2005;173(11):1335-40.

15. Hsu J, Price M, Huang J, Brand R, Fung V, Hui R, et al. Unintended consequences of caps on medicare drug benefits. N Engl $\mathrm{J}$ Med. 2006;354(22):2349-59. doi:10.1056/NEJMsa054436.

16. Hoffman J, Barefield FA, Ramamurthy S. A survey of physician knowledge of drug costs. J Pain Symptom Manag. 1995;10(6): 432-5. doi:10.1016/0885-3924(95)00018-T.

17. Weber ML, Auger C, Cleroux R. Knowledge of medical students, pediatric residents, and pediatricians about the cost of some medications. Pediatr Pharmacol (New York). 1986;5(4):281-5.

18. Heisler M, Wagner TH, Piette JD. Clinician identification of chronically ill patients who have problems paying for prescript ion medications. Am J Med. 2004;116(11):753-8. doi:10.1016/ j.amjmed.2004.01.013.

19. Shrank WH, Asch SM, Joseph GJ, Young HN, Ettner SL, Kholodenko Y, et al. Physician's perceived knowledge of and responsibility for managing patients' out-of-pocket costs for prescription drugs. Ann Pharmacother. 2006;40(9):1534-40. doi: 10.1345/aph.1H158.

20. The Canadian Press. Doctors' knowledge of drug prices could lower health costs: study (Online) 2007. http://www.cbc.ca/ health/story/2007/10/22/doctors-precriptionstudy.html. Accessed 22 Oct 2007.

21. Allan GM, Lexchin J, Wiebe N. Physician awareness of drug cost: a systematic review. PLoS Med. 2007;4(9):1486-96. doi: 10.1371/journal.pmed.0040283.

22. Taxis k, Schneeweiss S. Frequency and predictors of drug therapy interruptions after hospital discharge under physician drug budgets in Germany. Int J Clin Pharmacol Ther. 2003;41(2):77-82.

23. McCormack PME, Feely J. The impact of hospitalization on prescribing costs for medical patients returning to the community. Ir Med J. 1997;90(3):99-100. Apr May.
24. Ledwidge M, Travers B, Ryder M, Ryan E, McDonald K. Specialist care of heart failure improves appropriate pharmacotherapy at the expense of greater polypharmacy and drug-interactions. Eur $\mathbf{J}$ Heart Fail. 2004;6(2):235-43.

25. O'Connor PJ. Improving medication adherence. Challenges for physicians, payers, and policy makers. Arch Intern Med. 2006; 166(17):1802-4. doi:10.1001/archinte.166.17.1802.

26. Allan GM, Innes G. Family practice residents' awareness of medical care costs in British Columbia. Fam Med. 2002;34(2): 104-9.

27. Conti G, Delll”Utri D, Pelaia P, Rosa G, Cogliati AA, Gasparetto Al. Do we know the costs of what we prescribe? A study on awareness of cost of drugs and devices among ICU staff. Intensive Care Med. 1998;24(11):1194-8. doi:10.1007/s001340050744.

28. Horwitz LI, Kosiborod M, Lin Z, Krumholz HM. Changes in outcomes for internal medicine inpatients after work-hour regulations. Ann Intern Med. 2007;147(2):97-103.

29. Alexander GC, Casalino LP, Meltzer DO. Physician-patient communication about out-of-pocket prescription costs. JAMA. 2003;290(7):953-8. doi:10.1001/jama.290.7.953.

30. Feng CW, Dudley RA, Brook RH, et al. Elderly patients preferences and experiences with providers in managing their drug costs. J Am Geriatr Soc. 2007;55(12):1978-80.

31. Bellingham C. A simple discharge service that works. Pharm J. 2004;272:418.

32. Saunders SM, Tierney JA, Forde JM, Onorato AV, Abramson MH. Implementing a pharmacist-provided discharge counselling service. Am J Health Syst Pharm. 2003;60(11):1101, 1106, 1109.

33. Palmer HC, Armistead NS, Elnicki DM, Halperin AK, Ogershok PR, Manivannan S, et al. The effect of a hospitalist service with nurse discharge planner on patient care in an academic teaching hospital. Am J Med. 2001;111(8):627-32. doi:10.1016/S00029343(01)00976-7.

34. Office of the Attorney General. Florida prescription drug prices (Online). 2005. http://www.myfloridarx.com. Accessed 2 May 2008.

35. Michigan Department of Community Health (Online). 2006. MI prescription drug prices. http://www.michigandrugprices.com. Accessed 2 May 2008. 